\title{
Artigos
}

\section{MIGRACIÓN PERUANA, TRABAJO EN LA CONSTRUCCIÓN Y PRODUCCIÓN DEL ESPACIO EN LA CIUDAD DE CÓRDOBA, ARGENTINA}

\author{
Peruvian migration, job in the construction and production of space \\ in the city of Córdoba, Argentina
}

María José Magliano*

\begin{abstract}
Resumen. Este artículo se propone indagar en la articulación entre las trayectorias laborales en la construcción de varones peruanos en la ciudad de Córdoba (Argentina) y las formas de producción de los espacios donde se desenvuelve su vida cotidiana. En base a un trabajo de campo cualitativo sostenido en el tiempo en un barrio localizado en la periferia de esa ciudad, este estudio muestra el modo en que determinadas inserciones laborales funcionan como recursos estratégicos con los que cuentan las familias migrantes en pos de sostener su proyecto migratorio, asegurar su permanencia en el lugar de destino y resolver el derecho a la ciudad a través de la producción del espacio. En particular, los recursos que se obtienen a partir del trabajo en la construcción, habilitan diversas estrategias de reproducción de la vida tanto familiar como comunitaria en el marco de una ciudad cada vez más desigual.
\end{abstract}

Palabras clave: construcción, varones migrantes, producción del espacio, asentamientos informales, Córdoba.

Abstract. This article sets out to analyze the link between labor trajectories
in the construction sector of Peruvian migrants in the city of Cordoba
(Argentina) and the production of the space where these migrants manage
their daily life. Based on a qualitative field work sustained over time in a
peripheral neighborhood of that city, this paper shows the way in which
certain labor insertions work as strategic resources for migrant families in
order to reproduce their migration project, guarantee their permanence in

* Centro de Investigaciones y Estudios sobre Cultura y Sociedad (CIECS). CONICET y UNC. Buenos Aires, Argentina. 
destination places and resolve the right to the city through the production of space. In particular, the resources migrants obtain from construction work, allow different strategies of life reproduction, familiar and communal, in the framework of an increasing unequal city.

Keywords: construction sector, male migrants, production of space, slums, Cordoba.

\section{Introducción}

Los y las migrantes que han arribado en las últimas décadas a la Argentina se han dirigido principalmente hacia las áreas periféricas y relegadas de las grandes ciudades en un contexto de nuevas formas de pobreza y marginalidad urbana centradas en trayectorias laborales precarias e inestables ${ }^{1}$. En un escenario de creciente informalización de la vida cotidiana de las poblaciones migrantes, y a partir de un trabajo de campo sostenido en el tiempo en un barrio localizado en la periferia de la ciudad de Córdoba en Argentina, este artículo se propone indagar en la articulación entre las trayectorias laborales en la construcción de varones peruanos y las formas de producción de los espacios donde se desenvuelve su vida cotidiana. En tal sentido, en este estudio no nos detendremos en los modos en que se desarrolla la actividad de la construcción en sí misma, sino que nos focalizaremos en otro aspecto vinculado con esta trayectoria laboral, que ha recibido escasa atención, como es su relación con la autogestión y producción del espacio; es decir, reconstruiremos las formas en que los y las migrantes intervienen y transforman el lugar donde viven $^{2}$. El foco puesto en este aspecto permite analizar el modo en que las propias trayectorias laborales de los migrantes resuelven el derecho a la ciudad -entendida ésta como una más entre los lugares donde se entrecruzan diversos procesos transfronterizos para producir determinadas formaciones socioespaciales ${ }^{3}$ - a partir de reconocer que ciertos procesos productivos, como el caso de la construcción, activan una serie de alternativas y estrategias de acceso, circulación y asentamiento dentro de los espacios urbanos.

El escenario de estas indagaciones es Sabattini, un barrio ubicado en la periferia de la ciudad de Córdoba sobre terrenos fiscales del Estado nacional y habitado por unas 700 personas, en su mayoría de origen peruano. Casos como el de Sabattini muestran que el proceso de expansión urbanística

1 SASSEN, Saskia. Una sociología de la globalización. En Argentina, conclusiones similares emergen de los estudios de: GALLINATI, Carla. Vivir en la villa y luchar por la vivienda. O sobre una de las formas de ser migrante en la ciudad de Buenos Aires. Odisea. Revista de Estudios Migratorios, n. 2, 2015, p. 51-78; y VACCOTTI, Luciana. Migraciones, espacio y política Perspectivas teóricas para el abordaje del rol del Estado en la "lucha por la vivienda" (Ciudad de Buenos Aires, 2001-presente). Estudios Sociales Contemporáneos, n. 11, 2014, p. 38-50.

2 Para profundizar sobre la relación entre las migraciones limítrofes a la Argentina y la industria de la construcción, véase VARGAS, Patricia. Bolivianos, paraguayos y argentinos en la obra.

SASSEN, op. cit., p. 135. 
experimentada en Argentina desde la década del noventa, en un contexto de proliferación de los barrios cerrados y condominios en las grandes ciudades, ha configurado nuevas expresiones de "lo urbano", concebido como una construcción histórica y política ${ }^{4}$, acelerando una serie de tendencias que se venían desarrollando desde décadas anteriores. Entre ellas, el acceso al suelo se ha transformado en "un factor de inclusión o exclusión al derecho a la ciudad por parte de sus propios habitantes" ${ }^{\prime \prime}$, promoviendo y reproduciendo una ciudad cada vez más dividida, fragmentada y proclive al conflicto ${ }^{6}$. Así pues, en los últimos decenios, y asociado a trayectorias laborales fuertemente marcadas por la precarización y la inestabilidad, se produce un desplazamiento de los y las migrantes que llegan desde Bolivia, Paraguay y Perú hacia zonas específicas de la ciudad, en especial periféricas. Entre esas trayectorias, la construcción se convirtió en una inserción laboral de relevancia para los varones que han arribado desde esos países a las grandes ciudades argentinas.

En relación con la metodología, la permanencia sostenida en el tiempo en Sabattini, desde el año 2012 hasta el año 2016, resulta el pilar principal para las reflexiones que organizan este artículo. El inicio del trabajo de campo en ese espacio pretendía indagar en las trayectorias y experiencias migratorias de los varones y mujeres que, desde Perú, llegaron en el transcurso de las últimas dos décadas a la ciudad de Córdoba. Para ello, nos valimos centralmente de técnicas cualitativas de recolección de datos como la observación participante, las entrevistas informales mantenidas en el marco de nuestras visitas al barrio y las entrevistas en profundidad a mujeres y varones peruanos que allí residen. Para poder analizar la articulación entre migraciones, trabajo en la construcción y producción del espacio, en esta investigación priorizamos los registros etnográficos de nuestras visitas al barrio en el transcurso de los

\footnotetext{
WACQUANT, Loïc. Los condenados de la ciudad. Gueto, periferias y Estado, p. 21.

5 REBORD, Germán, MULATERO BRUNO, Daniela, FERRERO, Aurelio. Mercado informal del suelo urbano en Córdoba, p. 65. Otras investigaciones que han abordado los procesos de socio-segregación y urbanización en Córdoba son las de: BOITO, Eugenia, ESPOZ, Belén, SORRRIBAS, Patricia, Pensar los des-bordes mediáticos del conflicto: las ciudades-barrios como síntoma de la actual tendencia urbana de socio-segregación. Papeles del CEIC, v. 2012-1, n. 81, p. 1-40; MONAYAR, Virginia. Informalidad urbana y acceso al suelo. Acciones y efectos de la política habitacional en la ciudad de Córdoba-Argentina. Territorios, n. 24, p. 113-130; NUÑEZ, Ana, CIUFOLINI, Alejandra. Política y territorialidad en tres ciudades argentinas. Buenos Aires: El Colectivo, 2011.

6 HARVEY, David. Ciudades rebeldes. Del derecho de la ciudad a la revolución urbana.

7 Las entrevistas en profundidad fueron grabadas y constaron de más de un encuentro con los y las entrevistados/as. Las entrevistas informales fueron charlas que tuvimos con los/as vecinos/as en las distintas visitas al barrio. Esas charlas, en las cuales no se utilizó el grabador, fueron reconstruidas luego de la visita. Finalmente, los registros de la observación participante, que supone apuntar todo aquello que sucede durante el trabajo de campo, se realizaron también inmediatamente finalizaba la visita a Sabattini. Asimismo, es importante subrayar que los nombres de las personas como el del barrio fueron modificados para preservar su anonimato y resguardar su intimidad.
} 
cuatro años ininterrumpidos de trabajo de campo y las entrevistas informales realizadas con migrantes que se dedican a la construcción. Específicamente, el desarrollo de este artículo se sustenta en un total de 17 entrevistas informales realizadas a varones peruanos que trabajan en ese nicho laboral. Asimismo, incluiremos en el análisis 40 registros etnográficos de observación participante que hemos reunido durante el trabajo de campo. Tanto las entrevistas como los registros se recogieron durante nuestras visitas al barrio en el transcurso del trabajo de campo. En los dos primeros años, visitamos el barrio dos veces por semana, en general los días miércoles y sábados. Esas visitas duraban unas tres horas aproximadamente y, durante los años 2015 y 2016, se espaciaron convirtiéndose en semanales.

La relevancia de estas técnicas de recolección de datos se debe a que, como señala Bourgois, los lazos de confianza y los vínculos de larga duración con las personas que estudiamos y con el espacio donde esas personas subsisten cotidianamente, es clave para poder documentar la vida y las estrategias que despliegan los sectores marginados de las ciudades contemporáneas ${ }^{8}$. Nuestra presencia continua en Sabattini ofrece herramientas para pensar sobre el devenir de ese espacio, sobre su producción y autogestión a partir de una premisa central que reside en considerar al mercado de trabajo, al hogar y al barrio no como compartimentos estancos y aislados sino interconectados.

Además, este artículo recupera para su desarrollo los resultados del Censo de viviendas, hogares, población y sus características migratorias realizado en Sabattini en el año 20149 ${ }^{9}$. Este instrumento nos brinda elementos para comprender ciertas características socio-demográficas del barrio indispensables para los objetivos que orientan el texto. De acuerdo a los datos arrojados por dicho censo, la composición principal de la población según su origen nacional muestra un predominio de personas de origen peruano $(56,2$ por ciento); seguidas en orden de importancia proporcional por las de origen argentino (34,6 por ciento); boliviano ( 3,1 por ciento) y paraguayo $(2,7$ por ciento). La mayor parte de los habitantes del barrio son parejas jóvenes (tienen entre 20 y 40 años) que llegaron hace poco tiempo a la ciudad de Córdoba provenientes de Lima, aun cuando antes habían experimentado formas de migración interna en el Perú.

El conjunto de la información recopilada permite identificar, por un lado, las estrategias y los recursos con que cuentan las familias migrantes para sostener y consolidar su proyecto migratorio y para asegurar la reproducción

8 BOURGOIS, Philippe. En busca de respeto. Vendiendo crack en Harlem, p. 43.

9 La realización del censo resultó parte de un proyecto de investigación llevado adelante por parte de los integrantes del Programa de Investigación Multiculturalismo, migraciones y desigualdad en América Latina (CEA-UNC y CIECS-CONICET y UNC) y subsidiado por la Secretaría de Ciencia y Técnica de la Universidad Nacional de Córdoba (Argentina). 
de la vida; y, por el otro, las jerarquías y diferenciaciones que se establecen en los barrios informales y periféricos a partir del despliegue de esos recursos que descansan, en muchos casos, en las propias trayectorias laborales de quienes allí viven, como sucede con el trabajo en la construcción.

El artículo se organizará en tres apartados. En el primero, describiremos las características más salientes de la construcción como trabajo que ocupa a un número importante de migrantes sudamericanos en Argentina. En el segundo, indagaremos en la articulación entre la migración peruana en Córdoba y los procesos de llegada a ciertos espacios de la ciudad. Finalmente, en el tercer apartado nos concentraremos en la vinculación entre las trayectorias laborales en la construcción de los migrantes peruanos y la producción del espacio, atendiendo a dos dimensiones principales: la familiar y la comunitaria.

\section{La construcción como trabajo de "varones migrantes"}

En Argentina, la construcción se constituyó como un trabajo "atractivo" para la migración masculina limítrofe a partir de mediados del siglo XX en adelante, en especial para los migrantes que llegaban desde Bolivia y Paraguay a las principales ciudades del país ${ }^{10}$. En ese momento, y en el marco de la expansión del proceso de industrialización, las ciudades experimentaron una creciente demanda de actividades a realizar en las industrias que se abrían y en tareas relacionadas con la construcción y los servicios en los centros urbanos, producto del aumento de población ${ }^{11}$. Es en las décadas del sesenta y setenta del siglo pasado cuando se produce uno de los mayores incrementos de mano de obra migrante de origen limítrofe en la construcción, pasando del 9,8 por ciento al 20,9 por ciento $^{12}$. Para fines del siglo XX, esta actividad laboral se transformó también en un nicho privilegiado para los migrantes peruanos que arribaban al país en un contexto de crecimiento de la demanda de trabajo en este sector de la economía ${ }^{13}$.

Desde la década del noventa,

la participación del Producto Bruto Interno (PBI) de la construcción sobre el PBI total se ha mantenido casi constante oscilando entre un 5 por ciento y 6 por ciento. En el año 2002, en medio de la crisis socio-económica generalizada, la participación del PBI sectorial cayó hasta registrar un

\footnotetext{
${ }^{10}$ BENENCIA, Roberto. Apéndice: La inmigración limítrofe; VARGAS, op. cit.

${ }^{11}$ DOMENACH, Hervé, CELTON, Dora. La comunidad boliviana en Córdoba. Caracterización y proceso migratorio.

12 PANAIA, Marta. Los trabajadores de la construcción. Cabios y evolución del empleo en la industria de la construcción argentina (1947-1970), p. 34.

${ }^{13}$ La migración peruana hacia Argentina, que se ha caracterizado por ser un proceso fundamentalmente femenino y urbano, se intensifica en la década del noventa. Los varones peruanos que llegaron al país, muchas veces por reunificación familiar, se incorporaron principalmente en el sector laboral de la construcción.
} 
3,5 por ciento del PBI total. A partir de 2003 es cuando comienza a evidenciarse una recuperación que se mantiene durante el transcurso de aquella década ${ }^{14}$.

Bajo este escenario, a inicios del nuevo milenio los migrantes sudamericanos representaban el 5 por ciento del total de ocupados en el país; constituyendo el 11 por ciento del total de la mano de obra en el sector de la construcción para el caso de los varones y el 13 por ciento del total de la mano de obra en el trabajo doméstico remunerado para el caso de las mujeres ${ }^{15}$. En términos generales, los y las migrantes se han orientado hacia aquellas actividades del mercado peor remuneradas y con mayores niveles de inestabilidad laboral, poniendo de manifiesto el modo en que la etnicidad y el origen nacional funcionan como marcadores de posibilidades y de posiciones en la distribución de recursos en los países de destino ${ }^{16}$.

Dentro del sector de la construcción, que involucra desde las empresas constructoras hasta los sindicatos y los trabajadores, los migrantes integran una mano de obra que ocupa diferentes puestos. Esta diversidad depende de la capacitación que poseen, del tiempo de residencia en el lugar de destino y de las redes de contacto con las que cuentan. A partir de los resultados del trabajo de campo, y de manera similar a lo expresado por Vargas ${ }^{17}$, los migrantes peruanos que viven en Sabattini no solo cubren aquellos puestos más bajos de la obra sino que se los encuentra en distintas posiciones y asumiendo diferentes responsabilidades. Desde albañiles ocupados en empleos informales hasta encargados de obras de mayor envergadura que trabajan en relación de dependencia; desde personas recién llegadas sin ningún conocimiento en la materia, que comienzan a aprender el oficio a partir de obtener un primer trabajo por recomendación de un compatriota, hasta aquellas que poseen una formación importante, ya sea adquirida en su país de origen antes de migrar o en Córdoba, a partir de la experiencia que le ha otorgado la inserción en la propia actividad. En su conjunto, las redes sociales juegan un papel central a la hora de obtener un empleo que, entre los migrantes, se orienta principalmente al ámbito de la economía privada. Son justamente esas redes -que se materializan en contactos y recomendaciones, y que en ocasiones se

${ }^{14}$ RUGGIRELLO, Hernán. El Sector de la Construcción en perspectiva: internacionalización e impacto en el mercado de trabajo, p. 6. Para mayor información estadística respecto al rol de los migrantes sudamericanos en el ámbito de la construcción, véase: ARUJ, Roberto. Los trabajadores inmigrantes de la construcción: su aporte y significación. Buenos Aires: Organización Internacional para las Migraciones, 2012, p. 235-292; MONSALVO, Ana Paula. Los migrantes sudamericanos en el mercado de trabajo argentina: informalidad del empleo y brechas salariales. In La inmigración laboral de sudamericanos en Argentina. Buenos Aires: Organización Internacional del Trabajo, 2011, p. 131-156.

${ }^{15}$ MAGUID, Alicia. Migrantes sudamericanos y mercado de trabajo, p. 118.

${ }^{16}$ PEDREÑO CÁNOVAS, Andrés. Sociedades etnofragmentadas.

17 VARGAS, op. cit. 
activan en los lugares de origen previo a la migración-, las que orientan sus recorridos laborales y la circulación al interior de los espacios urbanos.

\section{Los migrantes peruanos y las llegadas a Sabattini}

La migración peruana hacia Argentina, que se ha caracterizado por ser un proceso fundamentalmente femenino y urbano, se intensifica en la década del noventa. Un amplio conjunto de las mujeres que llegaron en ese momento encabezó el proceso migratorio para insertarse laboralmente en el trabajo doméstico y del cuidado. Por su parte, los varones peruanos que llegaron al país, en muchos casos a partir de la reunificación familiar, se incorporaron principalmente en el sector laboral de la construcción.

Un porcentaje importante de los migrantes peruanos que arribaron en las últimas décadas a la Argentina residen en espacios similares a Sabattini, caracterizados por su precariedad e informalidad. En particular, la migración de quienes viven en este barrio es muy reciente, en todos los casos reconstruidos es posterior al año 2002. La llegada a Sabattini se dio luego de vivir unos pocos años en Córdoba, a partir de un proceso de "ocupación" de tierras dentro de las zonas urbanas, en general pertenecientes al Estado nacional, que hasta ese momento se encontraban deshabitadas. Los residentes de Sabattini no cuentan con ninguna documentación oficial que avale la posesión de los terrenos. Además, el barrio no posee gas natural, alumbrado público, cloacas y desagües. Las conexiones eléctricas son irregulares, determinado la mala calidad del servicio; y, desde el año 2014, el Municipio estableció un recorrido especial semanal para la recolección de residuos. Una de las principales problemáticas son las inundaciones de varios de los terrenos, debido a la falta de obras que permitan canalizar el agua cuando llueve. De acuerdo a las cifras arrojadas por el censo, más de la mitad de las viviendas del barrio (el 52,7 por ciento) sufre inundaciones cuando se acumula mucha agua producto de las lluvias y tormentas.

En este marco, Sabattini respondería a la categoría extendida de "asentamiento informal o precario"18. De acuerdo a un informe realizado en el año 2013, en la provincia de Córdoba se identificaron un total de 210 asentamientos informales similares a Sabattini con una cifra aproximada de 28.600 familias residiendo allí (ocupando el tercer lugar, luego de la provincia de Buenos Aires y la Ciudad Autónoma de Buenos Aires). En la ciudad de Córdoba, específicamente, el número de asentamientos informales al 2013 ascendía a 134, con un total estimativo de 21.300 familias residiendo en esos espacios ${ }^{19}$.

\footnotetext{
${ }^{18}$ Si bien Sabattini puede ser definido como un "asentamiento", en este trabajo elegimos hablar de "barrio", aunque informal, en tanto así es como lo denominan quienes allí viven.

19 TECHO ARGENTINA. Relevamiento de asentamientos informales. Buenos Aires: TECHO, 2013, p. 90. La organización no gubernamental latinoamericana Techo define conceptualmente
} 
La gran mayoría de los varones y mujeres que viven en Sabattini posee un empleo, principalmente en el ámbito informal. En el año 2014, al 68,9 por ciento de los varones y el 56,2 por ciento de las mujeres no se les realizaban los descuentos jubilatorios para la seguridad social, variable que puede tomarse como una aproximación a las condiciones de informalidad laboral que enfrenta un amplio conjunto de esta población. Tal como señala Monsalvo, la mano de obra migrante que llega de la región sudamericana presenta modalidades de inserción laboral específicas que se distinguen por una fuerte concentración en el sector informal del mercado de trabajo y por un patrón de inserción en algunas ramas de actividad ${ }^{20}$. Ese patrón se verifica también en Sabattini, donde la construcción (45 por ciento), el empleo doméstico remunerado (30 por ciento) y el trabajo textil (8 por ciento) representan las principales ocupaciones laborales de los varones y mujeres que allí residen, todas ellas marcadas por altas dosis de inestabilidad laboral.

En el caso de la construcción, la inestabilidad que caracteriza a esta ocupación, en especial para aquellos que se desempeñan de manera informal, es "compensada" por cierta facilidad para la obtención de trabajo, aun cuando sea siempre en condiciones precarias. Y esto puede vincularse al hecho de que, en el rubro de la construcción, ser migrante (boliviano, paraguayo o peruano) puede funcionar como "un recurso determinante a la hora de conseguir trabajo" ${ }^{\prime 21}$. Asimismo, es el trabajo en la construcción uno de los más valorados por los propios migrantes y sus familias. Este trabajo ha sido percibido por muchos migrantes, en especial aquellos que llegaron a Córdoba en el transcurso de la segunda mitad del siglo XX durante los procesos de urbanización e industrialización, como una contribución, buscando legitimar de esta manera su presencia en el lugar de destino ${ }^{22}$. Para los migrantes peruanos que conocimos en Sabattini, particularmente, la construcción es un nicho laboral especialmente valorado en un universo de escasas opciones laborales. Y esto se debe a que es un sector en donde efectivamente los migrantes "pueden obtener trabajo" $y$, en especial, uno que permita la subsistencia familiar y también un cierto reconocimiento social en el espacio del barrio.

a los asentamientos informales como "barrios informales que se constituyeron mediante distintas estrategias de ocupación del suelo, que presentan diferentes grados de precariedad y hacinamiento, un déficit en el acceso formal a los servicios básicos y una situación dominial irregular en la tenencia del suelo" (TECHO ARGENTINA, op. cit., p. 10).

20 MONSALVO, op. cit., p. 134.

21 VARGAS, op. cit., p. 30.

${ }^{22}$ MAGLIANO, María José. Los significados de vivir 'múltiples presencias': Mujeres bolivianas en Argentina, p. 165-195. Esto ha sido especialmente así para el caso de los migrantes bolivianos que llegaron a Córdoba en el transcurso de la segunda mitad del siglo XX en el marco de la expansión urbana e industrial. En sus relatos, estos migrantes enfatizan los "aportes" de la mano de obra boliviana en la construcción de edificios, rutas y diques en la ciudad capital y la provincia. 
Ese reconocimiento se asienta en las intervenciones directas que realizan los propios migrantes en el lugar, no solo en sus propias viviendas sino en barrio en su conjunto. El paisaje de Sabattini ha experimentado importantes transformaciones desde nuestra primera visita en julio del año 2012. Las casas de madera predominantes en aquel momento fueron dando paso a casas de otros materiales que los propios migrantes fueron construyendo, en general, durante los fines de semana. Y no solo eso, ciertos sectores del barrio sufrieron cambios producto de la intervención de los propios vecinos, sobre todo de aquellos que se dedican a la construcción. Así pues, mejoras en los ingresos y egresos, desagües para evitar las inundaciones, entre otras que veremos más adelante, han sido obras puntales en las cuales los migrantes han participado directamente. Ese "saber-hacer" que otorga la construcción a los varones peruanos, incluso aquellos que aprenden el oficio una vez que migran, no solo proporciona el sustento familiar, sino que también ofrece la posibilidad de mejorar el lugar donde se vive a través de la producción y autogestión del espacio. Y esto se vincula con el hecho de que, a través de este trabajo, los varones asumen la responsabilidad de la construcción y arreglo tanto de la vivienda familiar como de la infraestructura barrial, actuando directamente sobre el espacio, en un contexto de presencias "economizadas" y "adelgazadas" del Estado en estos lugares ${ }^{23}$. A partir de esta actuación, las familias migrantes buscan resistir y transformar la condición de "ilegitimidad" en el uso del espacio urbano, en el marco de formas concretas de acceso y apropiación asociadas a la "ilegalidad" de acuerdo a los parámetros establecidos por el Estado.

\section{El trabajo migrante y la producción colectiva del espacio}

El surgimiento de los llamados asentamientos informales en Córdoba se vincula con las dificultades que encuentran muchas familias de los sectores populares -tanto migrantes como nativas- para acceder a una vivienda. Ante esta situación, y como estrategia, las familias ocupan terrenos en precarias condiciones de infraestructura.

Hacia 1995, se inicia un proceso de diversificación del mercado informal, con la aparición de los llamados loteos fraudulentos o ilegales, en los que los responsables generan y venden lotes donde la normativa no lo permite. El éxito de esta modalidad se basa en que la necesidad habitacional lleva a la gente a no priorizar la existencia de comprobantes legales ni urbanos al momento de adquirir un terreno o vivienda ${ }^{24}$.

\footnotetext{
${ }^{23}$ DE MARINIS, Pablo. Derivas de la Comunidad: algunas reflexiones preliminares para una teoría sociológica en (y desde) América Latina, p. 89. De Marinis indica que la economización de las energías del Estado en espacios como Sabattini, lo cual remite a un nuevo formato "adelgazado" de actividad estatal, no implica retirada o desaparición.

${ }^{24}$ REBORD, MULATERO BRUNO, FERRERO, op. cit., p. 68.
} 
Este es el caso de Sabattini, donde a partir de un loteo en el año 2009 el predio comienza a poblarse con viviendas precarias que los mismos vecinos fueron levantando, en un primer momento de madera y, con el paso del tiempo, de materiales más sólidos. En esa primera etapa, muchas de las viviendas se construyeron con la ayuda de la Organización No Gubernamental Techo, tal como sucedió en otros barrios de Córdoba y del resto del país ${ }^{25}$. En promedio, los lotes poseen unos 200 metros cuadrados y las construcciones que se levantan no están habilitadas por ningún organismo público.

Nuestro argumento radica en considerar que la consolidación de Sabattini como barrio expresa el modo en que las familias de los sectores populares, donde se concentran las familias migrantes, descansan para su reproducción en el trabajo no remunerado tanto de las mujeres como también de los varones. Así, el esfuerzo por construir y recrear el barrio, por hacerlo habitable y duradero, se realiza comunitariamente -entendiendo lo comunitario como clave interpretativa para analizar las formas alternativas en que se gestiona la producción y la reproducción de la vida ${ }^{26}$ - a partir de las herramientas con que cada familia cuenta. Entre ellas, los conocimientos sobre la construcción de los varones se tornan centrales para pasar de viviendas de maderas y chapas a otras de ladrillo y cemento; y también para solucionar problemas de infraestructura que el barrio presenta y que el Estado (ya sea nacional como provincial y municipal) no resuelve. La convicción de los habitantes de Sabattini de que ese es su lugar en la ciudad -en parte por las constantes dificultades que enfrentaron desde su llegada a Córdoba en relación con los alquileres y la imposibilidad de obtener un lugar propio ${ }^{27}$ - es lo que impulsa el despliegue de una serie de esfuerzos individuales y colectivos en pos de mejorar las condiciones de vida familiares y barriales.

Los varones peruanos $-y$ no solo ellos- que llegaron a las ciudades argentinas en el transcurso de las últimas décadas muestran con sus trayectorias laborales no solo formas de etnización del mercado de trabajo a partir del origen nacional, la adscripción étnico-racial y la pertenencia de clase, que derivan en la constitución de nichos laborales con alta participación de población migrante; sino también el modo en que los recursos y herramientas que esas trayectorias habilitan, como en el caso de la construcción, se articulan con determinadas estrategias de gestión de la vida cotidiana en los lugares de destino. La producción del espacio a partir de la "toma" de un terreno y de la

\footnotetext{
${ }^{25}$ Esta organización, que mantiene sedes en distintos países de América Latina, viene trabajando en Argentina desde el año 2003.

${ }^{26}$ GUTIÉRREZ AGUILAR, Raquel, SALAZAR LOHMAN, Huáscar. Reproducción comunitaria de la vida. Pensando la transformación social en el presente.

${ }^{27}$ MAGLIANO, María José, PERISSINOTTI, María Victoria, ZENKLUSEN, Denise. Estrategias en torno a las formas de apropiación y organización del espacio en un 'barrio de migrantes' de la ciudad de Córdoba, Argentina.
} 
posterior construcción, en sus diversas etapas, de la propia vivienda familiar es una manifestación de esas estrategias.

\section{La dimensión familiar de la producción del espacio urbano}

En los más de cuatro años de presencia continua en Sabattini, fuimos testigos de una transformación significativa que experimentó el paisaje barrial: si en el año 2012 prevalecían las viviendas de madera, en los años subsiguientes la situación se fue modificando y las viviendas de material, aunque precarias algunas de ellas, predominaban. Para el año 2014, las construcciones que poseían ladrillo y cemento como materiales tanto del techo como de las paredes, eran las que encabezaban la tipología principal de las viviendas; seguidas por aquellas que poseían paredes de cemento y techos de chapas y, finalmente, por las de madera. Asimismo, los datos indican que, para aquel momento, el 63,5 por ciento de las viviendas poseía el baño dentro de las viviendas, seguido por el 31,7 por ciento que lo tenía fuera y un porcentaje no menor que no poseía (4,8 por ciento).

Las mejoras en las viviendas y en el barrio fueron producto de la intervención directa de sus habitantes. El caso de Mariano y Cecilia, ambos migrantes peruanos, es un buen ejemplo de la transformación a la que nos referimos. Su familia llega a Sabattini en el año 2009. Mariano se dedica a la construcción desde su arribo a Córdoba en el año 2006 y Cecilia al trabajo doméstico no remunerado y al trabajo comunitario (se ocupa de uno de los merenderos que existen en el barrio). Cuando los conocimos, en julio del 2012, su vivienda consistía en una construcción de madera que funcionaba, en ese momento, de habitación para ellos y sus dos hijas. Esa vivienda de madera fue levantada con la ayuda de la organización Techo, que comenzó a trabajar en Sabattini en los primeros años de construcción del barrio. Al igual que en otros espacios de la ciudad de Córdoba, y a partir de un modelo de intervención que consta de distintas etapas, Techo construyó varias casas en el barrio, consolidándose como una de las organizaciones con mayor presencia ${ }^{28}$.

Delante de esa vivienda de madera, Mariano construyó en el año 2011 una habitación de unos 25 metros cuadrados, junto con un baño, que oficiaba de cocina, comedor y, más adelante, de habitación matrimonial. Con el paso del tiempo, fue teniendo distintos usos, conforme se iban levantado (más tarde, con la ayuda de su yerno, también migrante peruano que se dedica a la construcción) otras habitaciones que se conectan con esa primera construida. La vivienda que Mariano construyó coincide con la tipología de las viviendas de las familias peruanas que habitan en los asentamientos informales de Córdoba.

${ }^{28}$ PERISSINOTTI, María Victoria. Procesos migratorios y experiencias políticas. Aportes teóricometodológicos desde la perspectiva etnográfica, p. 7. 
De acuerdo a investigaciones urbanísticas, y como observamos en la propia casa de Mariano y Cecilia, las viviendas de los y las migrantes peruanos/as tienden a ocupar la mayor parte del lote y a construir un mayor número de habitaciones ${ }^{29}$.

Mariano levantó la vivienda los fines de semana, sus "días libres" de trabajo en la obra. Durante la semana, su jornada laboral comienza a las 5:30 am y finaliza a eso de las $7 \mathrm{pm}$. Ante la imposibilidad de avanzar en la construcción de la vivienda familiar en la semana, Mariano se ocupó de ella especialmente los días sábado por la tarde, los domingos y los días feriados. Casos como este se replican en el barrio: aquellos varones migrantes dedicados a la construcción han intervenido directamente en sus propias viviendas y en el barrio en su conjunto. En esa intervención, se fueron dejando de lado las casas de madera dando paso a viviendas de materia ${ }^{30}$.

A pesar de que algunas mujeres participaron en la construcción de la vivienda, como parte de un proyecto que alcanzaba a todos los miembros de la familia; esta ha sido fundamentalmente una tarea masculina que involucró a los varones y, en algunos casos, a los jóvenes de la familia como parte de una doble estrategia: por un lado, colaborar con la construcción de la vivienda familiar y, por el otro, aprender el oficio e ir adquiriendo conocimientos para una posible futura inserción laboral en ese sector de la economía.

Ahora bien, las transformaciones en Sabattini a partir del "saber-hacer" de algunos de los migrantes que allí residen expresan, al mismo tiempo, fuertes jerarquizaciones al interior del barrio a partir de las propias trayectorias laborales. Esas trayectorias habilitan la posibilidad (o no) de hacer frente a las dificultades económicas y a la falta de acceso a créditos que enfrentan las familias que viven en los barrios informales y periféricos, a partir de la propia autogestión del espacio. Bajo este escenario, y en términos de recursos, ser albañil y dedicarse a la construcción no es lo mismo que ser trabajador textil, la otra ocupación que concentra a un importante número de varones peruanos. Tampoco es lo mismo, en términos de producción del espacio, vivir en familia que ser madre soltera y dedicarse al trabajo doméstico y del cuidado.

Lo que nos interesa resaltar es que los trabajos a los que acceden los migrantes determinan las posibilidades de producción del espacio, tanto familiar como comunitaria. La experiencia de Mariela y Héctor, quienes Ilegan a Sabattini en el año 2009, grafica esta situación. Desde su arribo a la ciudad,

${ }^{29}$ LEMMA, Martín, CÁCERES, Renzo, CÁCERES, Eduardo. Asentamientos urbanos periféricos en riesgo ambiental. El caso de Ampliación Nuestro Hogar III, p. 43.

${ }^{30}$ Los propios vecinos, varones y mujeres, expresan en sus relatos la importancia simbólica de las viviendas de material en contraposición a aquellas de chapa o madera. La noción de material para hacer referencia a las viviendas, es configurada por los y las vecinos/as como un anhelo en el marco de poder acceder a construcciones más fuertes y duraderas. 
en el año 2007, se dedican al trabajo textil, primero como empleados y luego como pequeños propietarios de un taller informal que funciona en su propia casa, ubicada en una de las calles principales del barrio. Como mencionábamos, además de la construcción para los varones y el trabajo doméstico remunerado para las mujeres, el trabajo textil ocupa al 8 por ciento de los y las migrantes que viven en Sabattini ${ }^{31}$. Desde que Ilegaron a Sabattini su vivienda sigue siendo la misma: una modesta construcción de madera levantada en su momento con la ayuda de la organización Techo. El deterioro de la vivienda con el paso de los años y el nacimiento de sus dos hijos, los llevaron a evaluar la posibilidad de una construcción de material. No fue sino hasta el año 2015 -cuando llega a Córdoba Diego, hermano de Mariela, quien está aprendiendo el oficio de albañil- que esta posibilidad puede llegar a concretarse. Con la ayuda de Diego, la idea es primero construir una habitación para el taller y, más adelante, el resto de la vivienda.

De acuerdo a lo que pudimos reconstruir, cada familia se ocupa de su propia vivienda familiar siendo poco usual que se trabaje en otras viviendas del barrio. Esto explica las dificultades de Mariela y Héctor para acceder a una vivienda de material y también de Juana, una migrante peruana que se dedica al trabajo doméstico remunerado. Juana vive sola con tres niños en primera infancia y edad escolar. Su casa, también de madera, sufre los embates del paso del tiempo sin poder hacer nada por mejorar las condiciones en las que tanto ella como su familia viven.

Estos ejemplos dan cuenta que la producción familiar del espacio no deja de responder a la lógica individualista como pauta de socialización principal que caracteriza a la ética neoliberal imperante ${ }^{32}$. Los lazos de solidaridad y las prácticas comunitarias entre quienes habitan Sabattini no necesariamente avanzan sobre el ámbito familiar, sino que esos lazos y prácticas se despliegan especialmente en aquello que es concebido como "público" y de uso común.

\section{La dimensión comunitaria de la producción del espacio urbano}

La producción del espacio no se agota en la dimensión familiar, expresada en la construcción y mejoramiento de la vivienda, sino que se extiende al espacio del barrio en su conjunto. La presencia "economizada" del Estado es suplida por el esfuerzo de aquellos vecinos y vecinas que cuentan con recursos para

\footnotetext{
${ }^{31}$ El trabajo textil se ha transformado en un "trabajo migrante" en Argentina desde comienzos de este siglo, en especial la última parte de la cadena productiva (corte y armado de la prenda) que se ha tercerizado. Las condiciones laborales y las formas de producción han propiciado el "abandono" de este sector de la economía por un amplio conjunto población "nativa". Para profundizar sobre este tema véase: BENENCIA, Roberto. El infierno del trabajo esclavo. La contracara de las 'exitosas' economías étnicas. Avá, n. 15, 2009, p. 43-72.

32 HARVEY, op. cit., p. 35.
} 
resolver algunas de las demandas que el barrio les impone. En este contexto, en Sabattini un grupo de migrantes peruanos comenzaron a pensar en algunas soluciones a su alcance para resolver problemas puntuales de infraestructura del barrio.

Fueron esos migrantes quienes construyeron, en el año 2015, una escalera de cemento que conduce a una de las entradas peatonales del barrio, aquella que colinda con las vías del ferrocarril, para facilitar tanto el ingreso como el egreso de las personas. Se ocuparon también de mejorar otros ingresos del barrio, nivelarlos y asfaltarlos, con el objetivo de facilitar el recorrido de los automóviles, en especial de las ambulancias en casos de emergencia. Fueron los mismos vecinos quienes colocaron, hace ya unos años, luces en las vías de ingreso para mejorar la iluminación y la seguridad, ya que Sabattini no cuenta con una red de alumbrado público. Todas estas intervenciones se realizaron en el marco de una doble preocupación: en primer lugar, hacer el barrio "transitable" para los vecinos, en especial los días de lluvia cuando gran parte del barrio se anega y para ciertos servicios públicos imprescindibles en caso de necesidad; y, en segundo lugar, mejorar la estética del barrio. "Queremos entre todos hacer el barrio más lindo", nos indicó Ramiro, un migrante peruano que se dedica a la albañilería, en un encuentro que mantuvimos en el año 2014 donde nos comentaba acerca de las mejoras que intentaban colectivamente hacer quienes, como él, residían en Sabattini (Ramiro, migrante peruano, 15/11/2014)33. Es decir que, ante una presencia "economizada" del Estado, el "saber-hacer" de los migrantes se materializa en la consolidación de un espacio marginal y periférico dentro de la ciudad a partir de un trabajo que es colectivo y es usufructuado por todos quienes allí viven.

Así como los varones buscan resolver comunitariamente la falta de infraestructura del barrio, las mujeres también cumplen con su parte en la consolidación colectiva de los barrios ubicados en los márgenes de la ciudad. Si bien no profundizaremos aquí sobre esta dimensión, es importante considerar que, vinculado también a sus propias inserciones laborales y reproduciendo ciertos roles de género, las mujeres peruanas en Sabattini despliegan una serie de prácticas y saberes en torno al cuidado comunitario con una fuerte impronta territorial (comedores y merenderos, por ejemplo), como un modo de poder asegurar la reproducción de la vida en esos espacios.

Este tipo de prácticas que involucran a las poblaciones que residen en los barrios informales y periféricos, expresan el sentido de lo colectivo para la producción de lo común, lo cual deviene de un proceso dinámico que

\footnotetext{
${ }^{33}$ Ramiro llega a Córdoba en el año 2008 con su mujer y su hijo (en Córdoba tuvieron dos hijos más). Una vez llegados a la ciudad, y como tantas otras familias, la necesidad los empuja a buscar un lugar para vivir a partir de la compra "irregular" de un lote en Sabattini. En su Perú natal, era maestro y vivía en la zona de la selva.
} 
gestiona y organiza la reproducción de la vida en su forma comunitaria ${ }^{34}$. Así pues, los lazos de solidaridad que se despliegan en espacios como Sabattini se sostienen, principalmente, en la necesidad de resolver cuestiones que atañen a la gran mayoría de quienes habitan el lugar.

Detrás de la producción comunitaria del espacio no hay una organización estable de personas, como sí la hay en los entramados comunitarios que se despliegan en torno al cuidado, sino que son grupos de vecinos -y no siempre los mismos- que se reúnen por cuestiones puntuales que afectan al barrio. Esto explica, entonces, que las coordinaciones respecto a las obras que se planifican y a las actividades que se desarrollan no sean fijas. En general, los propios vecinos otorgan un monto de dinero específico, en base a los cálculos que ellos mismos proyectan, para la compra de los materiales que involucra la realización de cada obra. Sin embargo, cuando esas obras cuestan una suma de dinero mayor a la que pueden afrontar, en muchos casos se solicitan "ayudas" externas, lo más usual es que éstas recaigan en las agrupaciones políticas que mantienen en ese momento una presencia activa en el barrio ${ }^{35}$.

Las decisiones que se toman en relación con el espacio público y su transformación pueden llegar a ser objeto de disputas entre los vecinos del barrio. Con esto queremos decir que lo comunitario y sus entramados no están exentos de conflicto, en especial cuando se trata de gestionar recursos que son siempre escasos. Así pues, los migrantes ensayan y redefinen en sus prácticas cotidianas formas de cooperación y autorregulación que les permiten avanzar sobre las diferencias internas que se presentan e intervenir sobre un espacio por fuera de la injerencia del Estado.

En este marco, el análisis de los recursos que otorgan ciertas trayectorias laborales, como la construcción, ofrece herramientas para reflexionar sobre aspectos que exceden la cuestión estrictamente vinculada al mercado del trabajo y su funcionamiento para involucrar la reproducción de la vida en su conjunto. Son esos recursos, reconvertidos en estrategias de subsistencia familiar y barrial, los que permiten resignificar la idea del derecho a la ciudad desde los márgenes.

\section{Conclusiones}

La apuesta principal de este artículo radicó en concebir a las trayectorias laborales y al mercado de trabajo como inextricablemente interconectadas con el hogar y el barrio. En este sentido, vimos cómo determinadas trayectorias

\footnotetext{
${ }^{34}$ GUTIÉRREZ AGUILAR, SALAZAR LOHMAN, op. cit., p. 37.

${ }^{35}$ Esas agrupaciones no tienen una presencia estable en el barrio. De hecho, desde el año 2012 pasaron varios actores políticos, tanto ONG como agrupaciones y movimientos políticos, por el barrio. Esa "inestabilidad" se debe a los propios conflictos que van emergiendo en territorios en constante disputa.
} 
laborales, en nuestro caso de varones migrantes, funcionan como recursos estratégicos con los que cuentan las familias migrantes en pos de sostener su proyecto migratorio y asegurar su permanencia en el lugar de destino. A partir de poner el foco en ciertas inserciones donde se concentran las poblaciones migrantes, como la construcción, pudimos observar el modo en que la producción de los espacios urbanos, en especial aquellos periféricos y marginales, descansan en las propias prácticas y necesidades de sus habitantes, muchas de las cuales van más allá de las normas establecidas por el Estado en torno a la "legalidad/ilegalidad".

El "saber-hacer" que otorga la construcción activa distintas estrategias de reproducción de la vida en el marco de una ciudad cada vez más desigual y excluyente. Bajo este marco, es importante reafirmar que ese "saber-hacer", que les permite a ciertos colectivos recrear las formas de acceso y permanencia en los espacios urbanos, no implica eximir al Estado del rol que le cabe y no cumple. Casos como Sabattini muestran los sentidos de las luchas cotidianas de los varones y mujeres migrantes que "día a día trans-forman y se empeñan en trans-formar su realidad social concreta y situada"36. Estas luchas forman parte de una dimensión clave del derecho a la ciudad, como es aquella que lo concibe como un derecho no solo a poder acceder a lo que ya existe sino, fundamentalmente, a poder cambiarlo ${ }^{37}$. En definitiva, la producción colectiva del espacio funciona como una respuesta, entre otras, que despliegan las familias migrantes en torno a la búsqueda de formas de reproducción de la vida más justas y dignas.

\section{Bibliografía}

BENENCIA, Roberto. Apéndice: La inmigración limítrofe. In DEVOTO, Fernando. Historia de la inmigración en Argentina. Buenos Aires: Editorial Sudamericana, 2003, p. 433-484.

BOURGOIS, Philippe. En busca de respeto. Vendiendo crack en Harlem. Buenos Aires: Siglo XXI editores, 2010.

CENSO DE VIVIENDAS, HOGARES, POBLACIÓN Y SUS CARACTERÍSTICAS MIGRATORIAS. Informe Final. Córdoba: Programa de Investigación Multiculturalismo, migraciones y desigualdad en América Latina, 2014.

DE MARINIS, Pablo. Derivas de la Comunidad: algunas reflexiones preliminares para una teoría sociológica en (y desde) América Latina. SINAIS. Revista Eletrônica - Ciências Sociais, n. 9, v. 1, 2011, p. 83-117.

DOMENACH, Hervé; CELTON, Dora. La comunidad boliviana en Córdoba. Caracterización y proceso migratorio. Córdoba: ORSTOM/Universidad Nacional de Córdoba, 1998.

${ }^{36}$ GUTIÉRREZ AGUILAR, SALAZAR LOHMAN, op. cit., p. 19.

37 HARVEY, op. cit. 
GUTIÉRREZ AGUILAR, Raquel; SALAZAR LOHMAN, Huáscar. Reproducción comunitaria de la vida. Pensando la transformación social en el presente. El Apantle. Revista de Estudios Comunitarios, n. 1, 2015, p. 15-50.

HARVEY, David. Ciudades rebeldes. Del derecho de la ciudad a la revolución urbana. Madrid: Ediciones Akal, 2013.

LEMMA, Martín; CÁCERES, Renzo; CÁCERES, Eduardo. Asentamientos urbanos periféricos en riesgo ambiental. El caso de Ampliación Nuestro Hogar III. Córdoba: Editorial de la Facultad de Arquitectura, Urbanismo y Diseño (UNC), 2016.

MAGLIANO, María José. Los significados de vivir 'múltiples presencias': Mujeres bolivianas en Argentina. Migraciones internacionales, n. 24, v. 7, 2013, p. 165195.

MAGLIANO, María José; PERISSINOTTI, María Victoria; ZENKLUSEN, Denise. Estrategias en torno a las formas de apropiación y organización del espacio en un 'barrio de migrantes' de la ciudad de Córdoba, Argentina. Estudios demográficos y urbanos, v. 29, n. 3, 2014, p. 513-539.

MAGUID, Alicia. Migrantes sudamericanos y mercado de trabajo. In La inmigración laboral de sudamericanos en Argentina. Buenos Aires: Organización Internacional del Trabajo, 2011, p. 109-130.

PANAIA, Marta. Los trabajadores de la construcción. Cabios y evolución del empleo en la industria de la construcción argentina (1947-1970). Buenos Aires: Ediciones del IDES, 1985.

PEDREÑO CÁNOVAS, Andrés. Sociedades etnofragmentadas. In PEDREÑO CÁNOVAS, Andrés; HERNÁNDEZ PEDREÑO, Manuel (coords.). La condición inmigrante. Exploraciones e investigaciones desde la Región de Murcia. Murcia: Universidad de Murcia, 2005, p. 75-106.

PERISSINOTTI, María Victoria. Procesos migratorios y experiencias políticas. Aportes teórico-metodológicos desde la perspectiva etnográfica. VIII Jornadas de Investigación en Antropología Social, Santiago Wallace, Facultad de Filosofía y Letras, Universidad de Buenos Aires, 27-29 de julio de 2016.

REBORD, Germán; MULATERO BRUNO, Daniela; FERRERO, Aurelio. Mercado informal del suelo urbano en Córdoba. Estudios Sociales Contemporáneos, n. 11, 2014, p. 63-75.

RUGGIRELLO, Hernán. El Sector de la Construcción en perspectiva: internacionalización e impacto en el mercado de trabajo. Buenos Aires: Aulas y Andamios, 2011.

SASSEN, Saskia. Una sociología de la globalización. Buenos Aires: Katz ediciones, 2007.

VARGAS, Patricia. Bolivianos, paraguayos y argentinos en la obra. Buenos Aires: Antropofagia, 2005.

WACQUANT, Loïc. Los condenados de la ciudad. Gueto, periferias y Estado. Buenos Aires: Siglo XXI Editores, 2007. 
Migración peruana, trabajo en la construcción y producción del espacio en la ciudad de Córdoba, Argentina

Recibido para publicación en 30.11.2016 Aceptado para publicación en 20.03.2017

Received for publication in November $30^{\text {th }}, 2016$

Accepted for publication in March 20 th 2017

ISSN impresso 1980-8585

ISSN eletrônico 2237-9843

http://dx.doi.org/10.1590/1980-85852503880005007 